\title{
Anreize zur Blutspende - ein wichtiges Thema
}

\author{
Friedger von Auer \\ Referat 115 «Blut und Blutprodukte, Sera und Impfstoffe, Gewebe», Bundesministerium für Gesundheit, Bonn, Germany
}

Das Thema Anreize zur Blutspende hat nicht nur in der Vergangenheit eine besondere Rolle gespielt, es ist auch heute stets präsent und ein Dauerthema und hat Eingang in nationale und internationale Regelwerke gefunden. Wie der Artikel von Alena M. Buyx [1] zeigt, wird das Thema erstaunlich vielschichtig und breit diskutiert. Neben Versorgungsaspekten spielen ethische, philosophische und wirtschaftliche Gesichtspunkte eine Rolle. Und natürlich verschieben sich die Gewichte der einzelnen Aspekte in der Diskussion je nachdem, wo die Diskussion geführt wird, ob in Europa, in den USA oder in Ländern der Dritten Welt. Demzufolge scheinen die Motive der Spenderinnen und Spender ganz unterschiedlich zu sein.

So kontrovers und engagiert die Diskussion auch geführt werden mag, so hat sie doch ein großes Ziel vor Augen, nämlich die Versorgung der Bevölkerung, wo immer in der Welt, mit hochwertigen Blutprodukten sicherzustellen. Die Blutspende ist kein Selbstzweck. Menschliches Blut wird benötigt, um mit den daraus hergestellten Blutprodukten Leben $\mathrm{zu}$ retten und schwerwiegende Krankheiten zu heilen. Die Blutspenderinnen und Blutspender stellen sich in den Dienst am Nächsten; sie erfüllen eine gesellschaftlich hoch geachtete Aufgabe. Deshalb weist das deutsche Transfusionsgesetz nicht nur den Blutspendediensten die Aufgabe der Versorgung mit Blut und Blutbestandteilen als öffentliche Aufgabe zu, sondern es würdigt auch die Spenderinnen und Spender als Menschen, die einen wertvollen Dienst für die Gemeinschaft leisten. Jedenfalls in den europäischen Ländern dürfte diese Einstellung das maßgebliche Motiv sein, Blut zu spenden.

Die ausreichende Versorgung der Bevölkerung mit Blutprodukten wird heute verstärkt unter dem Eindruck der älter werden Bevölkerung und dem zunehmenden Bedarf erörtert. Dieser faktische Druck der realen Verhältnisse zeigt, dass die Diskussion über Anreize für Blutspenderinnen und Blutspender nicht ausschließlich nach ethischen oder philosophischen
Gesichtspunkten geführt werden darf. Wer zum Beispiel die Frage der Unentgeltlichkeit der Blutspende ausschließlich mit ethischen Argumenten vertritt, gerät unweigerlich mit den täglichen praktischen medizinischen Notwendigkeiten in Konflikt. Wird z.B. in einem Notfall die Blutspende von gezielt ausgesuchten Personen benötigt (seltene Blutgruppe), dann muss gegebenenfalls auch ein finanzieller Anreiz zur Blutspende möglich sein. Obwohl das deutsche Transfusionsgesetz den Grundsatz der unentgeltlichen Spendeentnahme normiert hat, lässt es gleichwohl eine finanzielle Zuwendung im Ausnahmefall zu.

Nahezu unerschöpflich scheint die Diskussion über die Aufwandsentschädigung für die Blutspenderinnen und Blutspender zu sein, die das deutsche Transfusionsgesetz ausdrücklich zulässt. Sie kann auf ganz verschiedene Art und Weise gewährt werden, nämlich als attraktive Geschenke, Verlosungen, Gutscheine usw., aber auch als begrenzter pauschalierter Geldbetrag. Über die Zweckmäßigkeit und Vertretbarkeit der finanziellen Aufwandsentschädigung wird nach wie vor gestritten. Sie dient nicht nur der Erstattung von Fahrtkosten, sondern kann auch für den sonstigen Aufwand der Spenderinnen und Spender gewährt werden, und zwar differenziert nach der Spendeart. Mit dieser Regelung ist im Transfusionsgesetz eine pragmatische Lösung gefunden worden, die sich nach den Erfordernissen im Blutspendewesen richtet. Auch wenn ein finanzieller Ausgleich unterschiedlicher Höhe gewährt wird, handelt es sich nach dem Verständnis des Transfusionsgesetzes nicht um eine Bezahlung.

Unabhängig von der Diskussion über die Art und Höhe der Aufwandsentschädigung muss zweierlei immer klar sein: Es muss die Freiwilligkeit der Spende gewährleistet sein, und die Blutspenden müssen zum Schutz der Patientinnen und Patienten höchsten Qualitäts- und Sicherheitsansprüchen genügen. Diese Ansprüche sind in Regelungen der Europäischen Union und in Deutschland im Transfusionsgesetz

\section{KARGER}

Fax +497614520714

Information@Karger.de

www.karger.com (c) 2009 S. Karger GmbH, Freiburg

Accessible online at:

www.karger.com/tmh

Ministerialrat Friedger von Auer

Referat 115 «Blut und Blutprodukte, Sera und Impfstoffe, Gewebe»

Bundesministerium für Gesundheit

Heilsbachstraße 18, 53123 Bonn, Germany

Tel. +49 228 941-1150, Fax -4927

friedgervonauer@bmg.bund.de 
rechtlich abgesichert worden und werden in einer permanenten fachlichen Diskussion in den dafür gesetzlich vorgesehenen Fachgremien überprüft und weiterentwickelt. Dabei werden auch die Auswirkungen der Anreizsysteme für Blutspenderinnen und Blutspender berücksichtigt. Unter diesem Aspekt sind alle Untersuchungen und Anregungen zu solchen Anreizsystemen willkommen. Die Arbeit von Alena M. Buyx leistet hierzu einen willkommenen Beitrag.

\section{Literatur}

1 Buyx AM: Blood donation, payment, and non-cash incentives: classical questions drawing renewed interest. Transfus Med Hemother 2009;36:DOI 10.1159/000235608 